\title{
Modeling of a Thermosiphon to Recharge a Phase Change Material Based Thermal Battery for a Portable Air Conditioning Device
}

\author{
Rohit Dhumane Jiazhen Ling Vikrant Aute Reinhard Radermacher \\ Center for Environmental Energy Engineering, University of Maryland, College Park, 4164 Glenn L. Martin Hall \\ Bldg., MD 20742, USA \\ \{dhumane, jiazhen, vikrant, raderm\} @umd.edu
}

\begin{abstract}
Closed loop two phase thermosiphons have a broad range of applications due to their simplicity, reliability, low cost and the ability to dissipate high heat fluxes from minimal temperature differences. The present study focuses on one thermosiphon operation which solidifies a phase change material (PCM) based thermal battery for a portable air conditioner called Roving Comforter (RoCo). RoCo uses vapor compression cycle (VCC) to deliver cooling and stores the heat released from the condenser into a compact phase change material (PCM) based thermal battery. Before its next cooling operation, the PCM needs to be re-solidified. This is achieved by the thermosiphon, which operates within the same refrigerant circuitry with the help of a pair of valves. The molten PCM which acts as heat source affects the dynamics of the thermosiphon which in turn affects the solidification process. Thus the dynamics of both the PCM and thermosiphon are coupled. For accurate transient modeling of this process, the PCM model considers the solidification over a temperature range, variable effects of conduction and natural convection during the phase change and variable amounts of heat release at different temperatures within the temperature range of phase change. The paper discusses component modeling for this transient operation of thermosiphon and its validation with experimental data.
\end{abstract}

Keywords: Thermosiphon, Thermosyphon, Phase Change Materials

\section{Introduction}

A thermosiphon is an energy transfer device capable of transferring heat from a heat source to a heat sink over a relatively long distance, without the use of active control instrumentation and any mechanically moving parts such as pumps (Dobson and Ruppersberg, 2007). Thermosiphons are used in diverse applications like cooling of electronic components, light water reactors, solar water heating systems, geothermal systems, and thermoelectric refrigeration systems due to simple designs, simple operating principles and high heat transport capabilities (Franco and Filippeschi, 2011). Lack of moving component for pumping refrigerant also leads to higher reliability of the system. Thermosiphons may operate with single phase fluid or two phase fluid, may consist of a co-current or counter-current flow (Haider et al., 2002) and have open or closed loops (Benne and Homan, 2009). The counter-current thermosiphons are also referred to as heat pipes. Industrial applications typically involve the co-current thermosiphons and the term thermosiphon used henceforth in this paper, will refer to these co-current thermosiphons.

A closed-loop two-phase thermosiphon consists of a closed circuit of refrigerant tube filled with a working fluid (referred to as refrigerant in this paper) and oriented in a vertical plane. The refrigerant evaporates in the lower portion of the loop (called evaporator) due to a heat input. The resultant vapor then travels upwards through a vertical tube called as the riser to reach the condenser, where it rejects its latent heat. The condenser is located vertically above the evaporator and the condensed refrigerant from its outlet trickles down into the evaporator by gravity through the downcomer tube. The cycle repeats until the heat source is exhausted.

The current study is motivated by a need to understand the dynamics of a thermosiphon used to recharge the thermal battery of a portable air conditioning device called Roving Comforter (RoCo) (Du et al., 2016). The dynamic model is expected to aid the improvement of design and development of controls. A brief description of RoCo is given in the next section.

\section{System Details}

Traditional HVAC (Heating, Ventilation and Air Conditioning) systems consume significant amounts of energy to maintain a uniform temperature in the buildings within a narrow range, neither of which is necessary for delivering comfort (Hoyt et al., 2015). Personal conditioning systems like RoCo provide an opportunity to save the building energy by relaxing the building thermostat settings without compromising occupant thermal comfort.

RoCo uses vapor compression cycle (VCC) to deliver cooling for building occupants and stores the waste heat from the condenser in a compact PCM based thermal battery. The schematic of the two modes of operation of RoCo is shown in Figure 1. When the PCM is molten, the VCC operation is terminated. Due to the poor thermal conductivity of PCM, the molten PCM cannot solid- 


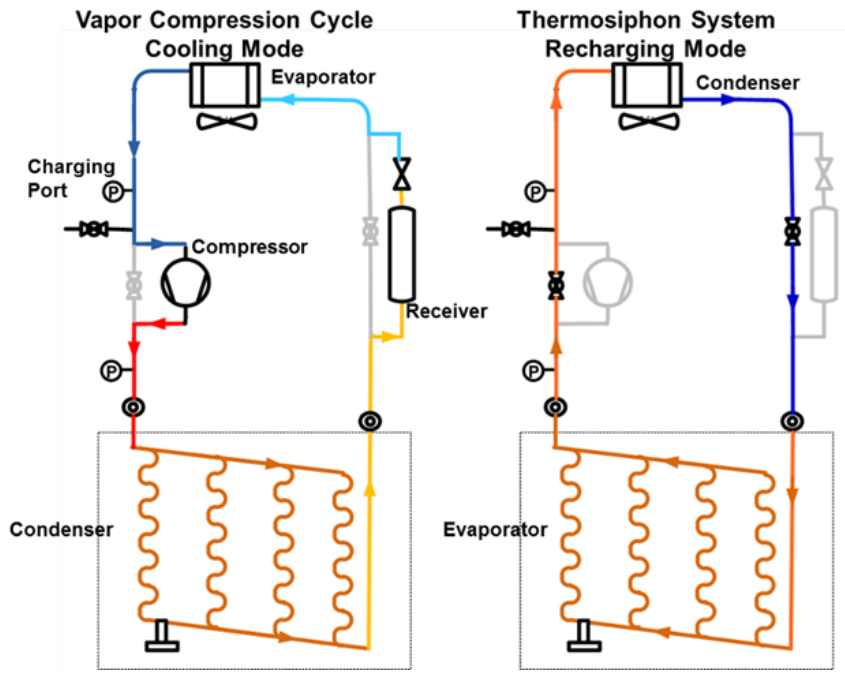

Figure 1. Schematic of two modes of operation of RoCo with the thermal battery marked in grey box

ify by itself within a reasonable time duration by rejecting heat to ambient air. Consequently, to enable a faster recharge of the thermal battery (i.e. PCM solidification), a thermosiphon is used. The thermosiphon operation is ideal in this situation because of its high rate of heat dissipation even from relatively small temperature differences between the heat source and the heat sink. The refrigerant circuitry is designed to enable a single direction flow of refrigerant. By operating a pair of valves, the refrigerant circuit switches from VCC circuit to thermosiphon circuit.

The PCM selected for the current application is paraffin-based, with the midpoint of its solidification temperature range at $35^{\circ} \mathrm{C}$. The temperature choice is based on a trade-off between two opposing factors. The temperature should be high enough so that the PCM does not solidify at typical room temperatures $\left(<26^{\circ} \mathrm{C}\right)$. At the same time, the temperature should also be low enough so that the condenser temperature for VCC operation is not very high. Higher condenser temperature leads to poor coefficient of performance (COP). Thus, a very narrow range of temperature range is applicable for the solidification temperature of PCM in RoCo. Paraffin based PCM is chosen because as a class paraffin is safe, reliable, predictable, less expensive and non-corrosive. It melts and freezes repeatedly without phase segregation and consequent degradation of its latent heat of fusion (Sharma et al., 2009). It crystallizes with little or no supercooling (Sharma et al., 2009). The only major disadvantage of paraffin based PCM is its low thermal conductivity. To address this issue, the thermal battery consists of helical coils of refrigerant tubing enclosed within PCM volume (See Figure 2). This arrangement enables higher surface area of heat transfer and better reach within the PCM volume.

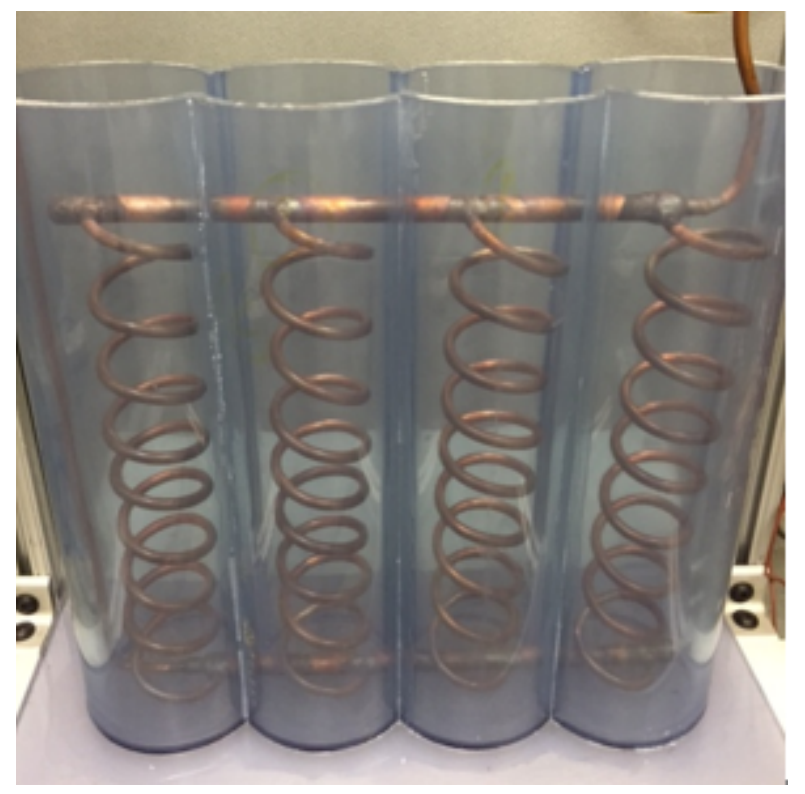

Figure 2. The thermal battery of RoCo in the experimental setup (Du et al., 2016)

\section{Model Development}

The system model for the thermosiphon consists of several components which are shown in Figure 3. The evaporator (See Figure 2) consists of four symmetric refrigerant circuits and to save computational effort, only one of them is modeled. The splitter and mixer components are used to scale the dynamic behavior of a single refrigerant circuit to the complete evaporator. The splitter divides refrigerant mass flow rates equally into four, while the mixer combines them. The refrigerant then flows into the riser, condenser, refrigerant tube and downcomer before flowing back to the evaporator. The refrigerant tube is a non-adiabatic flow passage for the refrigerant. The PCM blocks are connected to a tube control volume, which is a simple model of circular wall for pipes. The tube control volume component is also used to model the pcm container. Finally, the heat losses by natural convection and radiation from the pcm container to the ambient are incorporated. Detailed description of the component models is provided in this section.

\subsection{Phase Change Material}

Recall that the PCM Heat Exchanger (PCM-HX) consists of helical refrigerant tubes surrounded by PCM. The PCM solidification is a complex phenomenon due to the fact that the solid-liquid boundary moves depending on the rate of heat transfer and hence its position with time forms part of the solution (Zalba et al., 2003). The rate of heat transfer varies progressively during the phase change due to the varying effects of conduction and natural convection which depends on the state of PCM. Thus, the dynamics of PCM and thermosiphon are coupled. The helical nature of the refrigerant tube further increases the complexity by making the problem three-dimensional. 


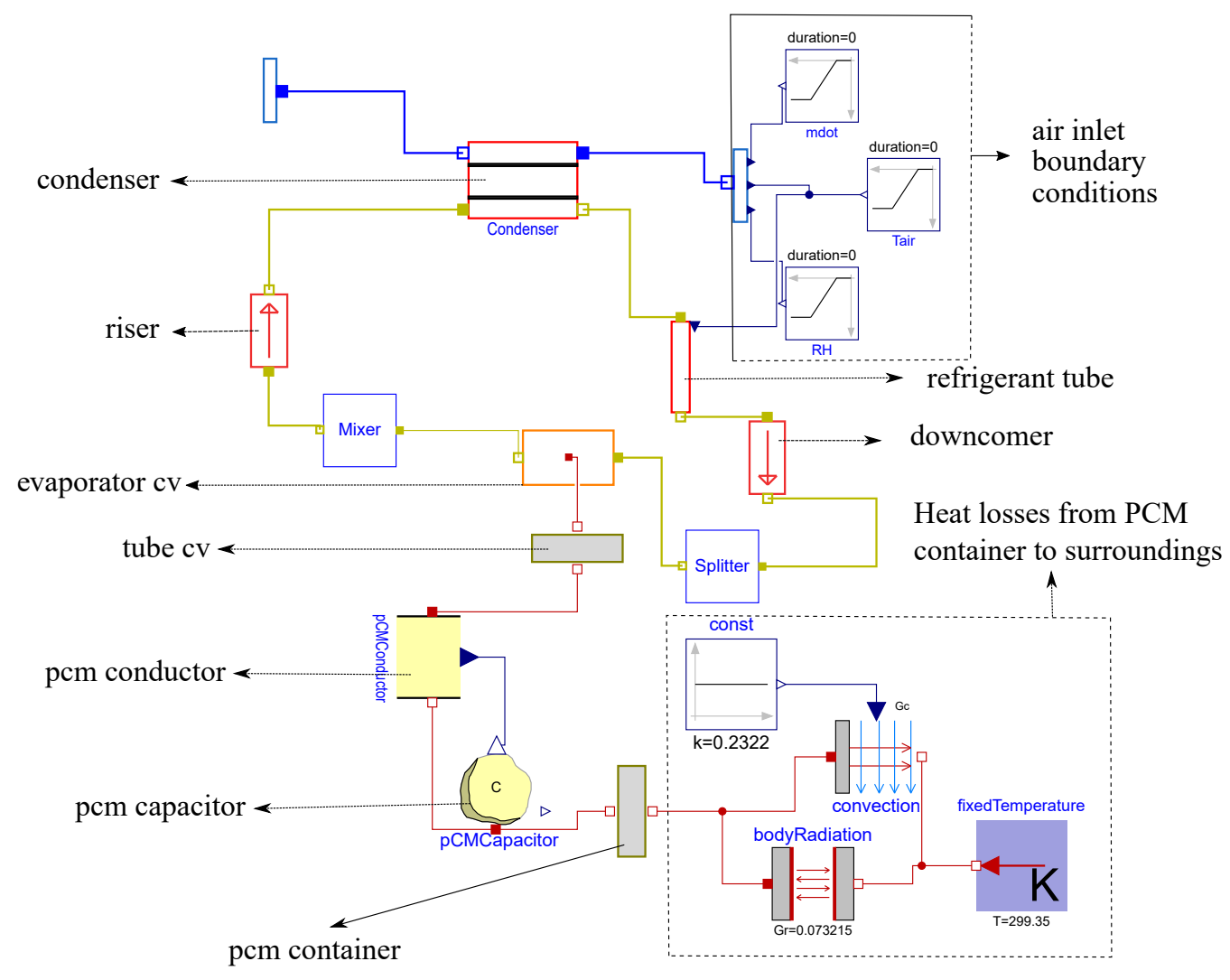

Figure 3. Schematic of System Model for Thermosiphon.

The model used in the current work is a trade-off for accuracy, complexity and usability. The PCM block is taken as a lumped control volume to eliminate the modeling of momentum equation for the molten PCM flow from natural convection. Two components are used to model PCM: $\mathrm{PCMC}$ nduct or to model the rate of heat transfer from the PCM and PCMCapacitor to model the PCM heat storage.

\subsubsection{PCM Capacitor}

The PCM-HX is the heat source for the thermosiphon and consequently dictates its dynamics. Very accurate description of its solidification is required. The energy equation applied to PCM control volume gives rise to:

$$
m_{p c m} \frac{d h}{d t}=\dot{Q}
$$

where, $m_{p c m}[\mathrm{~kg}]$ is the mass of PCM, $h\left[\mathrm{~J} \mathrm{~kg}^{-1}\right]$ is the specific enthalpy, $t$ [s] is the time and $\dot{Q}$ [W] is the rate of heat transfer.

The enthalpy method by Voller (1990) is used to model the energy equation. This method requires an input of enthalpy-temperature function of PCM solidification which is created using data from DSC readings of the PCM. This ensures accurate temperature prediction of the PCM state during solidification. The benefit of the enthalpy method is that it allows calculations on a fixed grid with implicit treatment of the phase change boundary.
Modelica.Blocks. Sources. CombiTable1D block is used for input of enthalpy-temperature profile.

The enthalpy-temperature profile is calculated as shown in equation (2)

$$
h(T)= \begin{cases}\int_{T}^{T_{A}} c_{s} d T, & \text { solid } \\ \int_{T_{B}}^{T_{A}} c(T) d T, & \text { two phase } \\ h_{f g}+\int_{T}^{T_{B}} c_{L} d T, & \text { liquid }\end{cases}
$$

$h_{f g}\left[\mathrm{~J} \mathrm{~kg}^{-1}\right]$ is the latent heat of melting, the PCM melts from temperature $T_{A}[\mathrm{~K}]$ to $T_{B}[\mathrm{~K}], c_{s}\left[\mathrm{~J} \mathrm{~kg}^{-1} \mathrm{~K}^{-1}\right], c(T)$ $\left[\mathrm{J} \mathrm{kg}^{-1} \mathrm{~K}^{-1}\right]$ and $c_{L}\left[\mathrm{~J} \mathrm{~kg}^{-1} \mathrm{~K}^{-1}\right]$ are specific heat capacities of PCM in the respective phases.

The melt fraction $(\lambda)$ of PCM is calculated from its enthalpy by the following equation:

$$
\lambda=\max \left(0, \min \left(1, \frac{h}{h_{l}}\right)\right)
$$

where $h_{l}\left[\mathrm{~J} \mathrm{~kg}^{-1}\right]$ is the enthalpy at the point where the PCM just turns liquid. The equation is simplified because of the fact that the enthalpy scale is defined as zero for the point where the PCM starts to melt. The melt fraction is made available for the PCM capacitor block through the Realoutput interface. 


\subsubsection{PCM Conductor}

The PCM Conductor block captures the variable effects of conduction and natural convection during the solidification of PCM. It calculates heat transfer coefficient as a function of melt fraction.

PCM Conductor block connects the refrigerant control volume of the condenser to the PCM Capacitor block. It extends Modelica. Thermal. HeatTransfer. Interfaces.

Element 1D block and provides for the heat flow, which is calculated using Combitable1D fitted function for heat transfer coefficient as a function of melt fraction. The Realinput interface is used to obtain melt fraction input from PCM Capacitor.

Table 1 contains the anchor points given to the CombiTable block used as input for the normalized heat transfer coefficient as a function of melt fraction. The constant value used to multiply the normalized function to obtain heat transfer coefficient (HTC) is $116 \mathrm{~W} \mathrm{~m}^{2} \mathrm{~K}^{-1}$. These numbers are obtained by matching the condenser pressure from simulation to the experiment since there are no correlations to capture the behavior in literature. Pal and Joshi (2001) discuss the heat transfer variation in the four regimes captured by Table 1 . The initial heat transfer occurs in a conduction dominated regime. Then there is a reduction in heat transfer coefficient with the appearance of small melt layer because the velocity of the liquid PCM due to buoyancy force is small. The melting then progresses to a convection dominated regime where the velocity of liquid PCM increases causing a higher rate of heat transfer. Finally, the magnitude of velocity decreases as the temperature in the molten PCM becomes more uniform with time due to natural convection stirring, leading to decreased buoyancy force for convection.

Table 1. Input table for PCM Conductor block.

\begin{tabular}{cc}
\hline Melt Fraction & Normalized HTC \\
\hline 0 & 1 \\
0.2 & 0.9 \\
0.4 & 1 \\
0.7 & 0.9 \\
1 & 0.8 \\
\hline
\end{tabular}

\subsubsection{PCM-HX Refrigerant Control Volume}

The PCM is modeled using a lumped control volume (CV) and accordingly a lumped control volume on the refrigerant side is required. These two CVs are connected using Modelica. Thermal. HeatTransfer. Interfaces. HeatPort interface.

The liquid refrigerant from the downcomer reaches the bottom header of the PCM-HX (See Figure 2). Then it absorbs heat from the PCM, vaporizes and rises up into the riser. The flow of refrigerant into and out from the $\mathrm{CV}$, is modeled using
Modelica.Fluid. Interfaces.FluidPort interface.

To define the state of refrigerant inside the lumped control volume two properties are required. The average density, $\rho_{\text {avg }}\left[\mathrm{kg} \mathrm{m}^{-3}\right]$ for the two phase refrigerant can be obtained as shown below:

$$
\begin{aligned}
V_{\text {tot }} & =V_{v}+V_{l} \\
m & =\rho_{v} V_{v}+\rho_{l} V_{l} \\
\rho_{\text {avg }} & =\frac{m}{V_{\text {tot }}}
\end{aligned}
$$

$V\left[\mathrm{~m}^{3}\right]$ refers to the volume of the refrigerant, $\mathrm{m}[\mathrm{kg}]$ its mass. The subscripts $v$ and $l$ refer to vapor and liquid phases while tot stands for total.

The pressure of the refrigerant is the average of the pressure at its inlet and outlet fluid ports.A medium record for the refrigerant is created and these thermodynamic properties are set to determine its state.

medium.d = rho_avg;

medium.p $=0.5 *($ port_a.p+port_b.p $)$;

The net pressure drop between the inlet and outlet ports is assumed to equal to the gravitational head offered by the refrigerant column.

The evaporator $\mathrm{CV}$ consists of liquid refrigerant with vapor escaping from the top after absorbing heat from the surrounding PCM. If the flow were to reverse, liquid refrigerant will leave out from the inlet port. Thus the stream variable of enthalpy in the fluid connectors are equated to the enthalpies of saturated liquid and vapor.

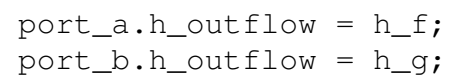

The heat flow term of the HeatPort is calculated by multiplying heat transfer coefficient by the product of surface area and temperature difference between HeatPort temperature and medium temperature.

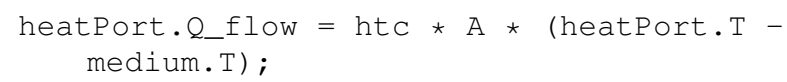

The two phase heat transfer coefficient for the refrigerant inside the helical coils is calculated first by using Schmidt (1967) correlation to obtain single phase liquid only heat transfer coefficient which is then used in Shah Chart correlation (Shah, 1982).

Finally, the mass and energy balance equations are written down and state transformations applied to update the values of pressure and enthalpy of the refrigerant $\mathrm{CV}$. This approach is pretty standard in two phase refrigerant system and is discussed in Tummescheit et al. (2000). The equations for energy, however, involve stream connector variations as described in Franke et al. (2009).

\subsection{Condenser}

The condenser in the system is a standard air to refrigerant heat exchanger. It is modeled using the heat exchanger developed by Qiao et al. (2015). The model neglects gravitational pressure drops. Thus, it can be visualized as if 
it is placed in a horizontal plane as opposed to vertical in the real case. However, the height of the condenser is small compared to the riser and gravitational effects can hence be ignored for model simplicity. The refrigerant side heat transfer coefficients for two phase flow are calculated using Shah (2016) correlation. The airside heat transfer coefficient is calculated using (Wang et al., 2000) correlation.

\subsection{Riser}

Recall that the riser is that portion of the refrigerant circuit where the refrigerant vapor rises from the evaporator into the condenser. The timescale over which its dynamics evolves is much faster than the heat exchangers. As a result, it is modeled as what is described as Flow Model in literature (Tummescheit et al., 2000). Only the momentum equation is used in the model and the mass and energy storage in the control volume are ignored. The momentum equation for riser contains balances for the pressure force, frictional force and gravitational force as shown in Equation 7 in which $L_{t}[\mathrm{~m}]$ is the length of the riser, $\frac{d \dot{m}}{d t}\left[\mathrm{~kg} \mathrm{~s}^{-2}\right]$ is the rate of change of refrigerant mass flow rate, $A\left[\mathrm{~m}^{2}\right]$ is the cross-section area for refrigerant flow in the tube, $p_{\text {in }}[\mathrm{Pa}]$ and $p_{\text {out }}[\mathrm{Pa}]$ are inlet and outlet pressures, $f$ is friction factor, $S[\mathrm{~m}]$ is the perimeter of the flow section of the tube, $\rho\left[\mathrm{kg} \mathrm{m}^{-3}\right]$ is refrigerant density and $g\left[\mathrm{~m} \mathrm{~s}^{-2}\right]$ is the acceleration due to gravity.

$$
L_{t} \frac{d \dot{m}}{d t}=A\left(p_{\text {in }}-p_{\text {out }}\right)-\frac{1}{2} \frac{\dot{m}^{2}}{\rho A^{2}} f S L_{t}+A \rho g L_{t}
$$

The friction factor equation incorporates laminar and turbulent flow regimes by merging Hagen-Poiseuille and Blasius equations. Both these equations are taken from Bergman and Incropera (2011).

\subsection{Downcomer}

The model for downcomer is similar to that of riser except for the momentum equation in which the direction of gravitational effects are reversed. The momentum equation for downcomer is shown in Equation 8.

$$
L_{t} \frac{d \dot{m}}{d t}=A\left(p_{\text {in }}-p_{\text {out }}\right)-\frac{1}{2} \frac{\dot{m}^{2}}{\rho A^{2}} f S L_{t}-A \rho g L_{t}
$$

\subsection{Heat Losses}

The PCM loses heat by natural convection and radiation with the surroundings. The heat loss by these modes are about $15-20 \%$ of the heat removed by the thermosiphon. For accurate prediction of solidification time, it is necessary to include these heat losses.

The PCM is contained in a PVC container. A simple Tube model of circular wall with one-dimensional heat conduction and capacitance lumped at arithmetic mean temperature is used. The equations for this model can be found in Modelica.Fluids.Examples.

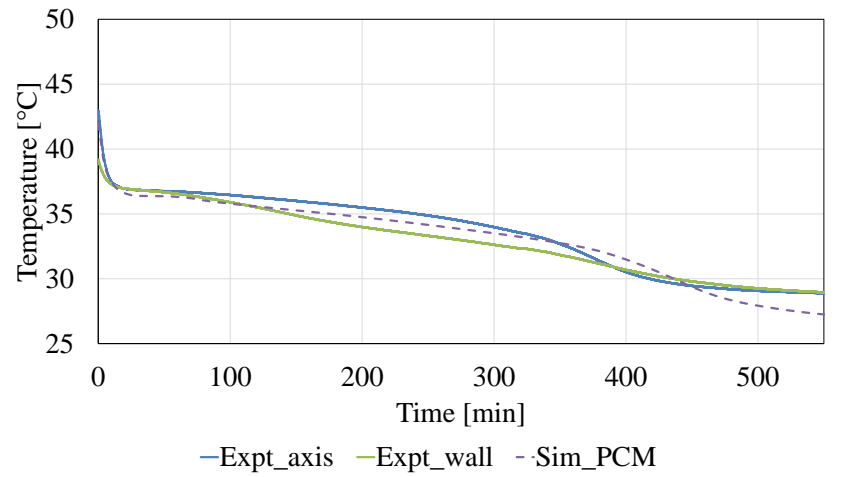

Figure 4. Comparison of PCM temperature prediction with experimental data

HeatExchanger. BaseClasses. WallConstProps.

The Tube model has two HeatPort interfaces, one of which is connected to the PCM Capacitor block. The second HeatPort is connected to a Modelica.Thermal. HeatTransfer.Sources. FixedTemperature block which has surrounding temperature, via Modelica. Thermal. Heat Transfer. Components. Convection and Modelica. Thermal. Heat Transfer. Components. BodyRadiation blocks to model the heat losses.

The heat transfer coefficient by convection from the container walls is obtained using Churchill and Chu (1975) correlation for natural convection for vertical plates. Radiation is calculated by taking a value of emissivity $\varepsilon=0.9$ for the material. The container is assumed to be a convex body in a large enclosure. The heat transfer coefficient from the top surface of the container is calculated using Lloyd and Moran (1974) correlation. However, this value is negligible in comparison to the net heat loss and omitted from the simulation.

\section{Results and Discussion}

Figure 3 shows the thermosiphon model with all the components described in the previous section. The boundary conditions and initial state points are provided using the experimental results from Du et al. (2016).

Figure 4 shows a comparison of PCM temperatures from the experiment with the model. There are two points from the experiment with subscripts axis and wall. The subscript axis refers to temperature probe near the PCM container wall while the subscript axis refers to temperature probe at the axis of the helical coil. The dotted line in the Figure 4 shows the lumped PCM temperature from the model. As can be observed, the overall prediction is good until roughly 430 minutes. The results deviate significantly from this point. This deviation can be attributed to the assumption of the adiabatic riser. In the experiment, there is heat loss from the refrigerant vapor as it passes through the riser leading to its condensation. The heat absorption from PCM drops significantly when the PCM is 


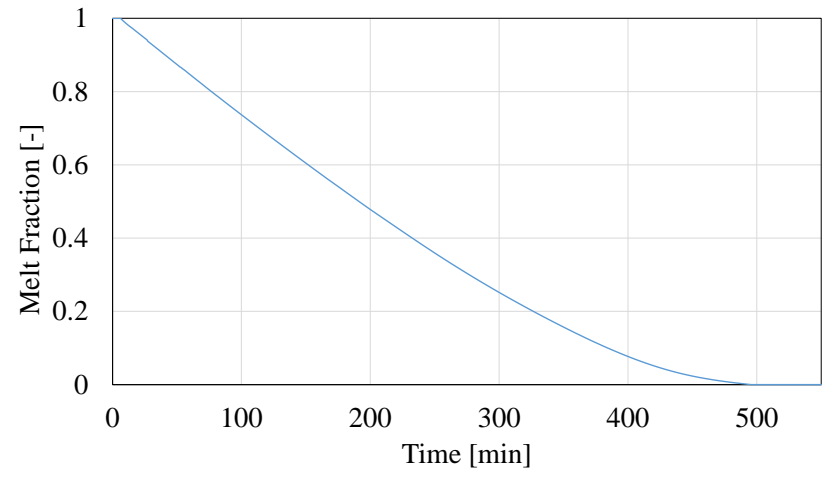

Figure 5. Percentage of PCM Molten with time

solidified. The model predicts a small rate of the mass flow rate at this point, but in reality there is no mass flow rate. The refrigerant vapor rises up but gets condensed and falls back down. This phenomenon is not captured by the model.

The recharge time calculated by the model is 489 minutes when the PCM fully solidifies (See Figure 5). However, $94 \%$ percent of PCM is solidified at the 400 minute mark. For a good overall cycle COP for RoCo, the thermosiphon can be operated for only 400 minutes and VCC operation started at this point.

Figure 6 shows the temperatures on the airside of the condenser. The prediction of air outlet temperature is slightly lower in the initial 20 minute interval. This can be attributed to the receiver present in the circuit which is filled with hot liquid refrigerant. For the setup, the receiver is sized in such a way that the downcomer is completely filled with liquid refrigerant. This results in larger thermal mass of refrigerant to be cooled.

\section{Conclusions}

A fully transient model for two phase closed loop thermosiphon is developed from first principles and used to study the dynamics of a thermosiphon used to recharge the thermal battery of a portable air conditioner. Equations to model various components of the thermosiphon

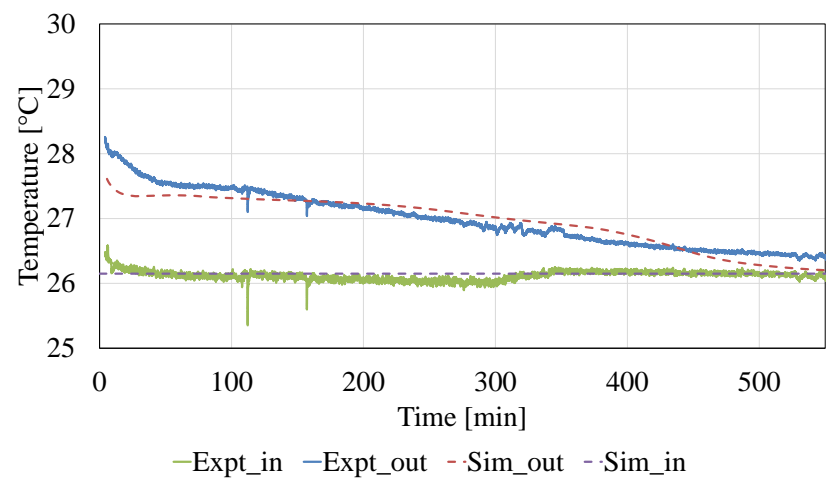

Figure 6. Comparison of air inlet and outlet temperatures at the condenser are discussed. The heat source of the thermosiphon is finite and the coupled dynamics is successfully predicted by the model. It is observed that solidifying 94\% of PCM is better than full solidification for better system COP. The model is expected to be an invaluable tool in designing the future versions of the portable air conditioning device with different requirements.

\section{Acknowledgment}

This research was supported by the Advanced Research Projects Agency - Energy (ARPA-E) with Award Number DE-AR0000530. We thank the members of Center for Environmental Energy Engineering (CEEE) and team members of the Roving Comforter Project for their support.

\section{References}

K S Benne and $\mathrm{K}$ O Homan. Transient Behavior of Thermosyphon-Coupled Sensible Storage with Constant Temperature Heat Addition. Numerical Heat Transfer, Part A: Applications, 55(2):101-123, 2009. doi:10.1080/10407780802552062.

Theodore L Bergman and Frank P Incropera. Introduction to heat transfer. John Wiley and Sons, Chichester, New York, 6 edition, 2011. ISBN 978-0470-50196-2.

Stuart W Churchill and Humbert HS Chu. Correlating equations for laminar and turbulent free convection from a vertical plate. International journal of heat and mass transfer, 18 (11):1323-1329, 1975. doi:10.1016/0017-9310(75)90243-4.

R T Dobson and J C Ruppersberg. Flow and heat transfer in a closed loop thermosyphon. Part I-Theoretical simulation. $J$. Energy South. Afr, 18:32-40, 2007.

Yilin Du, Jan Muehlbauer, Jiazhen Ling, Vikrant Aute, Yunho Hwang, and Reinhard Radermacher. Rechargeable Personal Air Conditioning Device. In ASME 2016 10th International Conference on Energy Sustainability collocated with the ASME 2016 Power Conference and the ASME 2016 14th International Conference on Fuel Cell Science, Engineering and Technology. American Society of Mechanical Engineers, 2016. doi:10.1115/ES2016-59253.

Alessandro Franco and Sauro Filippeschi. Closed Loop TwoPhase Thermosyphon of Small Dimensions: a Review of the Experimental Results. Microgravity Science and Technology, 24(3):165-179, 2011. doi:10.1007/s12217-011-9281-6.

Rüdiger Franke, Francesco Casella, Martin Otter, Michael Sielemann, Hilding Elmqvist, Sven Erik Mattson, and Hans Olsson. Stream Connectors - An Extension of Modelica for Device-Oriented Modeling of Convective Transport Phenomena. 43:108-121, 2009. doi:10.3384/ecp09430078.

S I Haider, Yogendra K Joshi, and Wataru Nakayama. A natural circulation model of the closed loop, two-phase thermosyphon for electronics cooling. Journal of heat transfer, 124(5):881-890, 2002. doi:10.1115/1.1482404. 
Tyler Hoyt, Edward Arens, and Hui Zhang. Extending air temperature setpoints: Simulated energy savings and design considerations for new and retrofit buildings. Building and Environment, 88:89-96, 2015. doi:10.1016/j.buildenv.2014.09.010.

JR Lloyd and WR Moran. Natural convection adjacent to horizontal surface of various planforms. Journal of Heat Transfer, 96(4):443-447, 1974. doi:10.1115/1.3450224.

Debabrata Pal and Yogendra K Joshi. Melting in a side heated tall enclosure by a uniformly dissipating heat source. International Journal of Heat and Mass Transfer, 44(2):375-387, 2001. ISSN 0017-9310. doi:10.1016/S0017-9310(00)001162.

Hongtao Qiao, Vikrant Aute, and Reinhard Radermacher. Transient modeling of a flash tank vapor injection heat pump system-part I: model development. International journal of refrigeration, 49:169-182, 2015. doi:10.1016/j.ijrefrig.2014.06.019.

Eckehard F Schmidt. Wärmeübergang und Druckverlust in rohrschlangen. Chemie Ingenieur Technik, 39(13):781-789, 1967. doi:10.1002/cite.330391302.

M M Shah. Chart correlation for saturated boiling heat transfer: equations and further study. ASHRAE Trans.;(United States), 88(CONF-820112-), 1982.

Mirza M Shah. Comprehensive correlations for heat transfer during condensation in conventional and mini/micro channels in all orientations. International journal of refrigeration, 67 : 22-41, 2016. doi:10.1016/j.ijrefrig.2016.03.014.

Atul Sharma, V V Tyagi, C R Chen, and D Buddhi. Review on thermal energy storage with phase change materials and applications. Renewable and Sustainable Energy Reviews, 13 (2):318-345, 2009. doi:10.1016/j.rser.2007.10.005.

Hubertus Tummescheit, Jonas Eborn, and Falko Wagner. Development of a Modelica base library for modeling of thermohydraulic systems. In Modelica Workshop 2000 Proceedings, pages $41-51,2000$.

V R Voller. Fast implicit finite-difference method for the analysis of phase change problems. Numerical Heat Transfer, 17(2):155-169, 1990. ISSN 1040-7790. doi:10.1080/10407799008961737.

Chi-Chuan Wang, Kuan-Yu Chi, and Chun-Jung Chang. Heat transfer and friction characteristics of plain fin-and-tube heat exchangers, part II: Correlation. International Journal of heat and mass transfer, 43(15):2693-2700, 2000. doi:10.1016/s0017-9310(99)00333-6.

Belen Zalba, Jose Ma Marin, Luisa F Cabeza, and Harald Mehling. Review on thermal energy storage with phase change: materials, heat transfer analysis and applications. Applied thermal engineering, 23(3):251-283, 2003. doi:10.1016/S1359-4311(02)00192-8. 